\title{
DIAGNÓSTICO DA REDE MUNICIPAL DE SAÚDE DE MACAÉ*
}

Isabela B. S.Tavares Amaral ${ }^{1}$

ORCID: https://orcid.org/0000-0003-4043-6020

Naiara Sperandio ${ }^{2}$

ORCID: https://orcid.org/0000-0002-9015-3849

Elenice Sales da Costa ${ }^{3}$

ORCID: https://orcid.org/0000-0002-3797-3784

Beatriz Dassie Carminatte ${ }^{4}$

ORCID: https://orcid.org/0000-0001-8470-2429

Diego Lima de Oliveira ${ }^{5}$

ORCID: https://orcid.org/0000-0003-1137-5740

Juliana Lourenço Barbosa ${ }^{6}$

ORCID: https://orcid.org/0000-0002-7754-5821

Luiza Lima Coutinho ${ }^{7}$

ORCID: https://orcid.org/0000-0002-7608-4509

Victoria Guitton Renaud Baptista de Oliveira ${ }^{8}$

ORCID: https://orcid.org/0000-0003-1121-9465

Recebido em: 11/06/2021.

Publicado em: 30/09/2021.

\footnotetext{
${ }^{1}$ Doutoranda em Saúde Coletiva: da área de Política, Planejamento e Administração em Saúde, pelo Instituto de Medicina Social da Universidade do Estado do Rio de Janeiro e Docente do curso de Graduação em Enfermagem da Universidade Federal do Rio de Janeiro- Campus Macaé- RJ, Brasil.

${ }^{2}$ Doutora em Ciência da Nutrição pela Universidade Federal de Viçosa e Docente do curso de graduação em Nutrição da Universidade Federal do Rio de Janeiro- Campus Macaé- RJ, Brasil.

${ }^{3}$ Enfermeira da Prefeitura Municipal de Macaé- RJ, Brasil, Coordenadora da Vigilância em Saúde, Especialista em Saúde da Família e Comunidade nos moldes da residência pelo Hospital Escola São Francisco de Assis/HESFA- UFRJ. ${ }^{4}$ Acadêmica de Enfermagem da Universidade Federal do Rio de Janeiro- Campus Macaé- RJ, Brasil.

${ }^{5}$ Acadêmica de Enfermagem da Universidade Federal do Rio de Janeiro- Campus Macaé- RJ, Brasil.

${ }^{6}$ Acadêmica de Medicina da Universidade Federal do Rio de Janeiro- Campus Macaé- RJ,Brasil.

${ }^{7}$ Acadêmica de Enfermagem da Universidade Federal do Rio de Janeiro- Campus Macaé- RJ, Brasil.

${ }^{8}$ Acadêmica de Medicina da Universidade Federal do Rio de Janeiro- Campus Macaé- RJ, Brasil.
} 


\title{
RESUMO
}

O presente estudo realizou um diagnóstico da rede de saúde pública do município de Macaé no estado do Rio de Janeiro (Brasil) com o objetivo de identificar a integração das redes de atenção à saúde (RASs), no período de 2019 a 2020. Trata-se de um estudo de abordagem quanti-quali. Foram utilizados questionários estruturados formulados a partir do instrumento proposto por Eugênio Vilaça Mendes que visa avaliar o estágio de desenvolvimento da(s) rede(s) de atenção à saúde. Participaram sete profissionais da RAS com experiência na gestão municipal. O município foi classificado segundo escore do instrumento de pesquisa, como tendo capacidade razoavelmente boa para operar a RAS. Foi observada a maior presença de "nós" nos componentes de Sistemas de Apoio e Sistemas Logísticos. Já as principais potências da rede mostraram-se presentes nos componentes de Atenção Primária à Saúde e Sistema de Governança e Modelos de Atenção à Saúde.

Palavras-chave: planejamento em saúde; políticas, planejamento e administração em saúde; serviços de saúde; Sistema Único de Saúde.

\section{DIAGNOSIS OF THE MUNICIPAL HEALTH NETWORK OF MACAÉ}

\begin{abstract}
A diagnosis of the public health network in municipality of Macaé in the state of Rio de Janeiro (Brazil) was carried out with the objective of identifying the integration of the health care network (HN), during the period of 2019 and 2020. Were used structured questionnaires, formulated from the instrument called: Questionnaire to assess the stage of development of the health care networks" proposed by Eugênio Vilaça Mendes. Seven selected professionals with experience in municipal management participated in this study. The municipality was classified, according to the score of their search instrument, as having good capacity to operate the HN. A greater presence of "knots" was observed in the components of Support Systems and Logistic Systems. On the other hand, the main strengths of the network showed themselves to be present in the components of Primary Health Care, the Governance System and Health Care Models.

Keywords: health planning; health's policies, planning and management; health services; Unified Health System.
\end{abstract}

\section{DIAGNÓSTICO DE LA RED MUNICIPAL DE SALUD DE MACAÉ}

\begin{abstract}
RESUMEN
El presente estudio realizó un diagnóstico de la red de salud pública en el municipio de Macaé en el estado de Río de Janeiro (Brasil) con el objetivo de identificar la integración de las redes de atención de salud (RAS), en el período de 2019 a 2020. Se utilizaron cuestionarios estructurados formulados a partir del instrumento propuesto por Eugênio Vilaça Mendes, denominado: Cuestionario para evaluar el estado de desarrollo de la(s) red(es) asistenciales. Participaron siete profesionales de RAS con experiencia en gestión municipal. El municipio se clasificó de acuerdo con la puntuación del instrumento de investigación, como de buena capacidad para operar el RAS. La mayor presencia de "nodos" se observó en los componentes de Sistemas de Soporte y
\end{abstract}


Sistemas Logísticos. Las principales competencias de la red, por su parte, se mostraron presentes en los componentes de Atención Primaria y Sistema de Gobernanza y Modelos de Atención en Salud.

Palabras-clave: planificación en salud; políticas, planificación y administración en salud; servicios de salud; Sistema Único de Salud.

\section{INTRODUÇÃO}

O Sistema Único de Saúde (SUS) configura uma engrenagem complexa cujo funcionamento adequado às necessidades da população prevê interlocução entre as diversas esferas da atenção à saúde, que vão desde o planejamento e gestão até a execução dos serviços em todos os níveis de assistência. Entretanto, tendo em vista as modificações que a organização dos serviços de saúde no Brasil passou ao longo desses mais de 30 anos de existência, torna-se cada vez mais importante a coerência entre a situação de saúde da população brasileira e o próprio SUS. Essa relação envolve inevitavelmente a ideia das redes de atenção à saúde (RASs) como uma forma de organização em sistemas integrados que viabilizem respostas eficazes, efetivas e seguras às condições de saúde da população no Brasil (MENDES, 2011).

Entendendo que a ideia de redes de atenção à saúde pode envolver linhas de cuidado, modelos de atenção, dentre outras diversas perspectivas, destaca-se que nos aspectos históricoconceituais, o primeiro relato que se pode correlacionar com o sistema de rede de atenção à saúde vem do relatório Dawson publicado em 1920 (OPS, 1964).

Esse documento desvela a proposta de organização de um sistema caracteristicamente regionalizado, com ênfase em um trabalho acessível a toda população e organizado segundo os níveis de complexidade e os custos do tratamento. Nele também observamos a valorização dos centros de saúde primários, apontados como porta de entrada do sistema e que de forma articulada e resolutiva fariam a interlocução com os outros níveis de atenção, setor secundário e hospitalar, com acompanhamento dos casos através de relatórios que nos remete ao que usamos hoje como referência e contrarreferência (MENDES, 2011).

Vale ressaltar ainda nesse sentido, que embora a premissa da organização de redes regionalizadas de saúde tenham constituído base para os debates das propostas de reforma do sistema de saúde brasileiro, o próprio rumo do processo de descentralização disparado na década 
de 90 , fez com que a ideia da constituição de redes tenha deixado de ser o eixo central em torno do qual se constituía a organização dos serviços (KUSCHNIR E CHORNY, 2010).

No Brasil apesar do SUS ter sido concebido legalmente a partir de 1990, com a lei orgânica da saúde, o adensamento desse debate é relativamente recente. Tem-se o marco com o Decreto 7508 de 2011, que conceitua rede de atenção à saúde como: “conjunto de ações e serviços de saúde articulados em níveis de complexidade crescente, com a finalidade de garantir a integralidade da assistência à saúde”'(BRASIL, 2011) e ainda, a Portaria No 3, de 28 de setembro de 2017, a qual institui as RASs e as conceituou como "arranjos organizativos de ações e serviços de saúde, de diferentes densidades tecnológicas, que integradas por meio de sistemas de apoio técnico, logístico e de gestão, buscam garantir a integralidade do cuidado” (BRASIL, 2017).

A lógica que permeia a ideia das RASs tem facilitado a integração e o processo de territorialização dos serviços, pois abrigam uma racionalidade nova de gestão e organização dos mesmos (VIANA et al, 2018). Salienta-se que uma rede integrada e regionalizada aponta para ganhos não apenas no sentido do acesso, mas também da qualidade dos serviços em saúde. Com o avanço da municipalização e regionalização ao longo do percurso de consolidação do SUS, o conhecimento acerca da organicidade do funcionamento local de cada cenário, pode apontar para o grau de capacidade de integração das redes de atenção à saúde ou para um estado de fragmentação das mesmas.

O presente trabalho toma enquanto objeto de pesquisa a rede municipal de saúde de Macaé e tem como objetivo realizar um diagnóstico dessa rede, quanto à sua capacidade operativa, visando identificar de que maneira a RAS municipal se situa entre um sistema fragmentado e uma rede integrada.

O presente artigo está divido da seguinte forma: após a apresentação do caminho metodológico trilhado pelos autores, os resultados são apresentados, primeiramente através de dados acerca dos participantes do estudo, e posteriormente, apresentam-se os resultados referentes a cada um dos sete componentes. Em seguida, aborda-se descritivamente ao final dos resultados, uma visão geral do diagnóstico da rede municipal de saúde. Por fim, os resultados apresentados são contextualizados e discutidos de forma mais qualitativa, sendo, logo após, tecidas as considerações finais. 


\section{METODOLOGIA}

Trata-se de um estudo transversal descritivo de abordagem quanti- quali que envolveu os profissionais que compõem a rede municipal de saúde de Macaé, Rio de Janeiro, no período compreendido entre outubro de 2019 e janeiro de 2020.

O município de Macaé, localizado na Região Norte Fluminense do Estado do Rio de Janeiro, tem seu cenário público de Atenção à Saúde, da qual depende cerca de $49 \%$ da população, composto por serviços de Atenção Primária, secundária e terciária à Saúde, geridos por meio da Secretaria Municipal de Saúde através de duas secretarias adjuntas: de Atenção Básica, e de Média e Alta Complexidade (LOURENÇO et al, 2019).

A equipe responsável pela condução da presente pesquisa é parte integrante do grupo pertencente ao projeto do Programa de Educação pelo trabalho para a saúde (PET) Saúde/Interprofissionalidade, foi composta por docentes e discentes dos cursos de enfermagem, farmácia, medicina e nutrição da Universidade Federal do Rio de Janeiro, além de preceptores do projeto que são profissionais de saúde atuantes na rede municipal de Macaé. Esta equipe foi previamente capacitada para compreensão dos conceitos pertinentes ao instrumento base de coleta de dados selecionado, proposto por Eugênio Vilaça Mendes (MENDES, 2011). Este formulário é aplicado nas dimensões da população, da atenção primária, dos pontos de atenção secundária e terciária, dos sistemas de apoio, dos sistemas logísticos, do sistema de governança e do modelo de atenção à saúde.

Há estudos que abordam a validação desse instrumento em versões adaptadas para linhas específicas como saúde bucal (LEAL; WERNECK; BORGES-OLIVEIRA, 2017) e atenção materno-infantil (SCHILLER et al, 2020), ou ainda, versão específica para avaliar a capacidade da Atenção Primária à Saúde (APS) em coordenar as RASs (RODRIGUES et al, 2014). No entanto, neste estudo, a aplicação do instrumento objetivou avaliar o estágio de desenvolvimento das redes de atenção à saúde no âmbito do município em questão, para trazer ao conhecimento como as mesmas estão organizadas e permitir identificar um contínuo que pode situar-se entre um sistema fragmentado até uma rede integrada, exatamente conforme a finalidade original proposta por (MENDES, 2011). 
Foi elaborado ainda pela equipe um glossário anexado com termos técnicos presentes nas perguntas do instrumento e que talvez fugissem do entendimento dos voluntários, já que alguns termos são específicos da referência utilizada.

Cada componente do formulário é composto do seguinte quantitativo de questões: componente da População com 14 questões; componente da APS com 19 questões; componente dos Pontos de Atenção à saúde secundários e terciários com 43 questões; componente dos sistemas de apoio com 15 questões; o componente dos Sistemas logísticos com 16 questões; o componente do sistema de Governança da Rede com 14 questões, e o componente dos modelos de atenção à saúde com 53 questões, totalizando 174 questões. Cada questão deveria ser respondida com um valor numérico correspondente que varia de 0 a 3 , sendo:

0 (zero): corresponde à não existência do que está sendo perguntado;

1 (um): existe o que está sendo perguntado, mas com um funcionamento básico ou incipiente;

2 (dois): existe o que está sendo perguntado, com um funcionamento razoavelmente bom, mas insuficiente;

3 (três): corresponde à sua existência em operação de forma ótima.

Ao final de cada formulário, havia um espaço de preenchimento opcional para que os sujeitos pudessem comentar ou acrescentar informações que julgassem pertinentes.

Foram realizados contatos com profissionais da gestão municipal de saúde e da própria rede de serviços, (participantes do Projeto PET-Saúde), para identificar aqueles cuja expertise pudesse contribuir com o conhecimento das dimensões abordadas no instrumento proposto. Foram identificados sete profissionais. A partir de então, elaborou-se formulários específicos, estruturados e de autopreenchimento distribuindo uma parte das questões do formulário para cada sujeito de pesquisa, de acordo com sua expertise.

Foram incluídos sete profissionais em regime estatutário que compõem as Redes de Atenção à Saúde no município de Macaé, desde a gestão até a assistência direta e que aceitaram participar da pesquisa. Os critérios de exclusão consistiam em: serem profissionais, que durante a etapa de coleta de dados estavam em período de férias, licença maternidade ou outros 
afastamentos e aqueles que apontaram qualquer impedimento ou impossibilidade de responder às perguntas do questionário.

O início da etapa de coleta de dados se deu mediante contato prévio com os serviços de saúde, que participaram do PET - Saúde/Interprofissionalidade, dentre eles a própria secretaria municipal de saúde, para que fosse agendado junto aos profissionais contatados, uma data para a entrega do formulário a cada um deles individualmente. Neste contato eram expostas todas as etapas da pesquisa, a relevância da mesma, os riscos e benefícios. Também foi garantido todo o sigilo das informações coletadas. Explicava-se para os participantes da pesquisa como se dava esse preenchimento e a partir daí era combinada uma data para o recolhimento desse questionário. A pesquisa foi realizada seguindo a Resolução 466/12 que fala dos critérios éticos de pesquisas envolvendo seres humanos, preservando e garantindo a confidencialidade e a privacidade dos indivíduos envolvidos na pesquisa, assegurando assim sua proteção de imagem e sua não estigmatização, não prejudicando os mesmos em nenhum aspecto pessoal e/ou profissional. Obtevese a aprovação segundo o número CAEE: 17725219.4.0000.5699. Cada profissional, que aceitou participar, assinou o Termo de Consentimento Livre e Esclarecido (TCLE).

Para a análise dos dados, procedeu-se à soma dos pontos recebidos para cada elemento presente nos componentes avaliados, resultando em um total para cada componente e, por fim, um resultado final correspondente ao somatório do total de cada componente. O resultado final foi interpretado com base na seguinte tabela de pontuação:

Tabela 1 - Escore para interpretação dos resultados

\begin{tabular}{|c|c|}
\hline Escore & Interpretação \\
\hline 0 a 130 & $\begin{array}{c}\text { Incapacidade para a operação de redes: sistema } \\
\text { fragmentado }\end{array}$ \\
\hline 131 a 260 & $\begin{array}{c}\text { Capacidade básica para operar redes: rede de } \\
\text { atenção à saúde incipiente }\end{array}$ \\
\hline 261 a 390 & $\begin{array}{c}\text { Capacidade razoavelmente boa para operar redes: } \\
\text { rede de atenção à saúde avançada }\end{array}$ \\
\hline 391 a 522 & $\begin{array}{c}\text { Capacidade ótima para operar redes: redes } \\
\text { integradas de atenção à saúde }\end{array}$ \\
\hline
\end{tabular}

Fonte: Mendes, 2011 
O autor Mendes (2011) cita que devem ser destacadas as questões que obtiveram pontuação entre 0 e 1, pois isso auxilia na identificação de possíveis nós críticos da rede e a partir deles se pode configurar um plano de ação para as correções necessárias. Portanto, no presente artigo, foram destacados nos resultados as pontuações 0 e 1 , de modo que pudessem fornecer um panorama de quais dos blocos avaliados apresentaram os maiores desafios, e enfatizadas aquelas que atingiram pontuação máxima, como exemplificação dos pontos mais fortalecidos da Rede analisada. Além disso, a pontuação total atingida de cada bloco foi comparada com a pontuação máxima possível potencialmente alcançável, para identificação das dimensões avaliadas que apresentaram maiores "hiatos" e aquelas que mais se aproximaram de um desempenho potencialmente alcançável a partir do instrumento utilizado.

\section{RESULTADOS}

Os achados estão divididos entre o perfil dos entrevistados, e os componentes avaliados da rede, de forma a compor uma compreensão mais alargada das fragilidades e das potências de cada uma dessas dimensões, especificamente dentro da realidade da Rede Municipal de Saúde de Macaé. Segue-se por fim, um tópico que aborda as principais considerações acerca do diagnóstico realizado, trazendo a pontuação total do município no instrumento de avaliação.

\section{População do estudo}

O perfil dos participantes da pesquisa foi composto por 3 voluntários do sexo masculino, que correspondem a 42,7\% dos entrevistados e 4 voluntárias do sexo feminino, correspondendo a $57,1 \%$. Dentre eles, a mediana de idade foi de 43 anos, tendo o mais jovem deles 33 anos e o mais velho 57 anos.

Compuseram a população do estudo as seguintes categorias profissionais: 1 administrador, 2 enfermeiros (sendo um deles também assistente social), 1 farmacêutico, 1 fisioterapeuta, 1 nutricionista e 1 médico.

Com relação ao tempo de experiência de trabalho no âmbito da gestão que os participantes apresentaram, em média os voluntários têm 10 anos e 5 meses, seja dentro ou fora do município de Macaé. O menor tempo apresentado foi de 4 anos e o maior de 20 anos. Especificamente nos 
serviços de Macaé a média de anos na gestão é de 4 anos e 8 meses, encontramos entre eles com menor tempo 1 ano e 3 meses e com maior tempo 9 anos. $O$ tempo de experiência acumulada entre serviços de saúde e de gestão em saúde, a média é de 13 anos e 5 meses, sendo o mínimo encontrado de 3 anos e o máximo 30 anos.

Quanto à apresentação dos resultados encontrados, a organização foi feita com base na estratificação dos componentes apresentados no instrumento utilizado para coleta de dados na presente pesquisa, conforme descrito a seguir.

\section{Componente 1 - População}

A população é o primeiro elemento das RASs, que precisa ser organizada em territórios sanitários, em famílias, e é cadastrada por riscos sociosanitários (MENDES, 2011). Sendo assim, a população de uma RAS deve ser registrada corretamente nos sistemas de informação disponíveis e subdividida em subpopulações.

Tal componente alcançou a pontuação total de 64,3\%, sendo o escore máximo de 42 pontos e foi obtido 27 pontos, assim foram identificadas entre as 14 questões fechadas, a pontuação máxima (escore 3) apenas em duas questões, e a pontuação mínima (escore 1 e 0 ) em duas questões também.

Evidenciou-se que a população está subdividida razoavelmente bem quando se trata dos riscos sociais, perfil demográfico, fatores de risco e por condições de saúde estabelecidas.

Além disso, os dados também apontaram que existe um processo de territorialização que dispõe de um Plano Diretor de Regionalização (PDR), e ele corresponde à sua existência operando de forma ótima. Entretanto o PDR na sua estruturação está insuficientemente relacionado a incorporação dos princípios de: contiguidade territorial, subsidiariedade econômica e social, economia de escala, relações entre escala e qualidade, identidade cultural, fluxos vários, fluxos assistenciais, acesso e oferta de pontos de atenção à saúde secundários e terciários.

Por fim podemos destacar que os nós críticos estão relacionados à estruturação operacional da RAS que é incipiente em relação às necessidades da população adscrita, e essa mesma população segue não sendo cadastrada pelo segmento de unidade familiar. 


\section{Componente 2 - Atenção Primária à Saúde (APS)}

A APS passou por uma renovação nos anos 2000 que a torna mais abrangente e integrada no sistema de saúde, como aponta a OMS (2008). Nesse sentido assegura-se que a APS é o nó intercambiador das RASs no qual se espera que haja um grau de articulação com a rede que faça desse nível um verdadeiro centro de comunicação que coordena os fluxos e contrafluxos do sistema de atenção à saúde, assumindo um papel de coordenadora do cuidado, guiando a população adscrita dentro do sistema de saúde através da equipe de saúde da família. (MENDES, 2011).

No presente estudo observou-se que este componente atingiu uma pontuação máxima de $70,2 \%$ sendo o escore máximo de 57 pontos, sendo obtido através da coleta de dados 40 pontos. O principal nó crítico apontado estava relacionado à falta de interação entre as equipes da APS com os níveis de atenção secundária e terciária. Além disso, é importante ressaltar ainda que apesar de haver Conselho municipal de saúde funcionante, o município não apresenta conselhos locais de saúde nas unidades da APS para potencialização do controle social do SUS.

Entretanto é possível destacar o resultado de que existe um funcionamento razoavelmente bom na APS, no que diz respeito ao papel claro da sua equipe e sua responsabilização pela população agrupada, de acordo com as linhas-guias, ou seja, as linhas de cuidado estruturantes dos processos de cuidado de acordo com as condições de saúde e o incentivo para a população usar a APS como porta de entrada da RAS.

Também apresentaram funcionamento razoavelmente bom: a clareza das relações definidas entre a APS e os demais níveis de atenção à saúde da RAS; a operação com sistemas de informação suficientes para registrar e disponibilizar as informações clínicas, epidemiológicas e gerenciais; a organização para prestação de cuidados às condições agudas e/ou intercorrências das condições crônicas de acordo com a classificação de risco; a organização para prestação de cuidados às mulheres, crianças, adolescentes e idosos por equipe multiprofissional segundo as linhas-guias e seu acompanhamento e ainda, prontuário clínico estruturado por famílias, com utilização rotineira dos instrumentos de abordagem familiar.

É possível destacar também em relação à APS, que ela opera de forma ótima. Os dados apontam que as equipes na APS possuem responsabilidades bem definidas e que se articulam de forma rotineira com as organizações da comunidade, apontam ainda para existência de vínculo estreito e contínuo entre as equipes, os usuários e seus familiares; capacitação dos Agentes Comunitários de Saúde (ACS) para busca ativa e seu papel de articulação entre a comunidade e o 
serviço. Além disso, foi apontado ainda que, a APS é utilizada como requisito para acessar outros níveis de atenção à saúde da RAS.

\section{Componente 3 - Os pontos de atenção à saúde secundários e terciários}

A atenção secundária e terciária vista como ponto da Rede de Atenção à Saúde, ou seja, como parte dela, possibilita que suas ações estejam integradas às redes, facilitando a interação da APS com os pontos de atenção secundária, voltadas às microrregiões sanitárias e os pontos de atenção terciária, voltadas para as macrorregiões sanitárias, sendo este uma perspectiva inovadora que vai de encontro com o modelo biologicista, centrado nas especialidades médicas (MENDES, 2011).

O componente de atenção à saúde secundária e terciária alcançou 55,8 \% do total de pontos (72 de 129 pontos), sendo identificada pontuação máxima em 27,9 \% das questões (o que representou 12 de 43 perguntas) e pontuação mínima em 18,6\% das questões (representando 8 de 43 perguntas).

Foram identificados como pontos positivos da atenção secundária e terciária no município, ou seja, aqueles que apresentaram pontuação máxima: oferta de cirurgia ambulatorial; oferta de leitos de longa permanência; serviços hospitalares de nível terciário para traumas e urgências e emergências clínicas, principalmente cardiovasculares; maternidade de alto risco operante de forma integrada com a APS; serviços hospitalares terciários, para cuidados aos cânceres, principalmente de colo de útero e mama e unidades para cuidados às doenças renais operando de forma integrada com a APS.

Destacaram-se ainda operação ótima dos serviços ambulatoriais especializados e hospitalares às mulheres e às crianças, para os cuidados à hipertensão e às doenças cardiovasculares e serviços ambulatoriais especializados às doenças bucais, sendo apontada operação de forma integrada com a APS, o que vai necessariamente de encontro ao que foi apontado na avaliação do componente anterior, onde a articulação, do ponto de vista da APS com os níveis secundário e terciário, foi o principal nó crítico identificado. Aponta-se ainda nesse sentido, que o componente 3 também mostrou funcionamento incipiente dos instrumentos operacionais de contrarreferência para APS, sendo o fortalecimento destes, um dos caminhos possíveis para alcançar de forma mais eficaz, a integração entre APS e os demais níveis assistenciais. 
Por outro lado, dentre os "nós" da rede que apresentaram a pontuação mínima destaca-se: a existência e articulação de uma casa de apoio à gestante e puérpera com a maternidade de alto risco; a existência e operacionalização de serviços ambulatoriais especializados em urgência e emergência com a APS; ao estabelecimento de proximidade entre os serviços ambulatoriais e hospitalares com a APS por meio de linhas-guia bem definidas; e a oferta de hospitais/dia e de centros de enfermagem (nursing homes).

\section{Componente 4 - Sistemas de Apoio}

Ao analisar os Sistemas de Apoio da Rede, entendidos como lugares institucionais onde serviços comuns são prestados a todos os pontos de atenção à saúde, diversos desafios foram destacados. Este componente atingiu apenas 28,9\% da pontuação máxima.

Um total de $80 \%$ das respostas (12 de 15) classifica o que estava sendo perguntado como inexistente ou com um funcionamento básico ou incipiente, o que traduz uma avaliação bastante negativa deste componente.

Os aspectos identificados como os principais "nós", fizeram referência direta a: atenção farmacêutica, conciliação de medicamentos, manejo de adesão aos tratamentos medicamentosos, farmacovigilância, sistema estruturado de exames de imagens, protocolos clínicos de orientação para indicação dos exames de imagem e análises clínicas, bem como a descentralização das coletas das amostras, dentre outros aspectos relacionados à assistência farmacêutica. Foi considerado como existente em operação de forma ótima apenas o armazenamento e mecanismo de aquisição de insumos farmacêuticos.

\section{Componente 5 - Sistemas Logísticos}

Os Sistemas Logísticos têm a função de garantir uma organização de fluxos e contrafluxos de informações, produtos e pessoas nas RAS, por meio de soluções tecnológicas (MENDES, 2011). Este componente alcançou apenas 39,6\% da pontuação potencialmente alcançável.

Os resultados apresentados neste componente tornaram possível identificar muitos entraves e desafios, que corresponderam a 62,5\% das respostas (10 de 16) com escore representado entre 01, ou seja, inexistente ou com um funcionamento básico ou incipiente. Parte significativa dos nós 
críticos identificados refletiram situações que corroboram com os achados da análise do componente da APS no que tange a falta de integração com os demais níveis da rede, como por exemplo: a não possibilidade de agendamento direto de atendimentos ou intervenções eletivas na atenção ambulatorial especializada ou na atenção hospitalar pela APS.

Todavia, pontos positivos foram destacados também, em 5 perguntas recebendo a pontuação 3, ou seja, existente em operação de forma ótima, foram esses: a existência do número ou cartão com identificação para cada usuário, a regulação das urgências e emergências com médico regulador permanente, o transporte de urgência e emergência prestado por ambulâncias de suporte básico e avançado, o transporte para procedimentos eletivos com veículos adequados e transporte de amostra de exames e análises clínicas.

\section{Componente 6 - Sistema de Governança}

O Sistema de governança das RAS é o arranjo organizativo que permite a gestão dos componentes da Rede, com vistas a obter bons resultados no campo sanitário e também econômico para a população adscrita (MENDES, 2011). As perguntas sobre o Sistema de Governança alcançaram $66,7 \%$ do total da pontuação máxima alcançável no componente (28 pontos de 42).

Aquelas que receberam uma melhor avaliação, sendo destacado como existente em operação de forma ótima, traduziram as respostas de 6 das 14 perguntas. Dentre estas, ressalta-se aquelas que versam sobre sistema de contratualização dos prestadores de serviço, gestão financeira eficaz, eficiência técnica e alocativa, plano estratégico de curto, médio e longo prazo, foco na maximização da eficiência, e incentivos financeiros alinhados com os objetivos das RAS. Em contrapartida como inexistente ou com um funcionamento básico ou incipiente foram apontadas em 4 respostas das 14 perguntas; a presença de um sistema de acreditação da RAS, a avaliação dos usuários com relação as RAS, a estrutura organizacional de governança e o compartilhamento de missão, valores e visão pelos participantes da RAS.

\section{Componente 7 - Modelos de Atenção à Saúde}

O Modelo de atenção à saúde pode ser entendido como um sistema capaz de organizar a 
operacionalização das RASs a partir da articulação entre "a população e suas subpopulações estratificadas por risco, os focos das intervenções do sistema de atenção à saúde e os diferentes tipos de intervenções sanitárias [...]” (MENDES, 2011, p. 211).

No componente "modelos de atenção à saúde", foram alcançados 69 pontos em um total de 159 pontos, o que equivale a $43,4 \%$ da pontuação máxima potencialmente atingível no componente, formado por 53 perguntas. Os pontos positivos da rede identificados, ou seja, aqueles que atingiram pontuação máxima no escore, correspondem a 13,2\% das perguntas realizadas (7 de 53). Dentre esses aspectos destacam-se enquanto resultados apresentados que: o plano de cuidado de cada paciente é realizado por uma equipe multiprofissional; há instrumentos operacionais que organizam as ações de prevenção de condições de saúde, os plano de cuidados são realizados por uma equipe multiprofissional em conjunto com o usuário, a gestão da condição de saúde envolve a educação em saúde dos usuários, por meio da atuação de profissionais com conhecimento e por meio de tecnologias e instrumentos construídos e avaliados com base em evidências.

Em relação aos "nós" enfrentados pela rede, que apresentaram pontuação 0 ou 1 no escore, corresponderam a um total de 32 das 53 perguntas. Dentre essas fragilidades foram identificadas dentre outras: a falta de definição clara de um modelo de atenção à saúde que seja conhecido na APS e nos pontos de atenção primária e secundária, que proponha ações de autocuidado apoiado e ações relativas aos recursos da comunidade. Foi identificado também à falta de organização de fluxos e contrafluxos das pessoas na APS, nos pontos de atenção secundários e terciários e nos sistemas de apoio, com base nas linhas-guia, além da falta de revisão periódica e outras fragilidades no âmbito das mesmas.

\section{Visão geral do Diagnóstico da rede municipal de saúde}

Como forma de melhor visualizar o desempenho dos componentes apresentados, segue abaixo a figura 1 como imagem ilustrativa da pontuação total alcançada em cada um deles, em comparação a máxima potencialmente alcançável: 
Figura 1 - Pontuação obtida x pontuação máxima

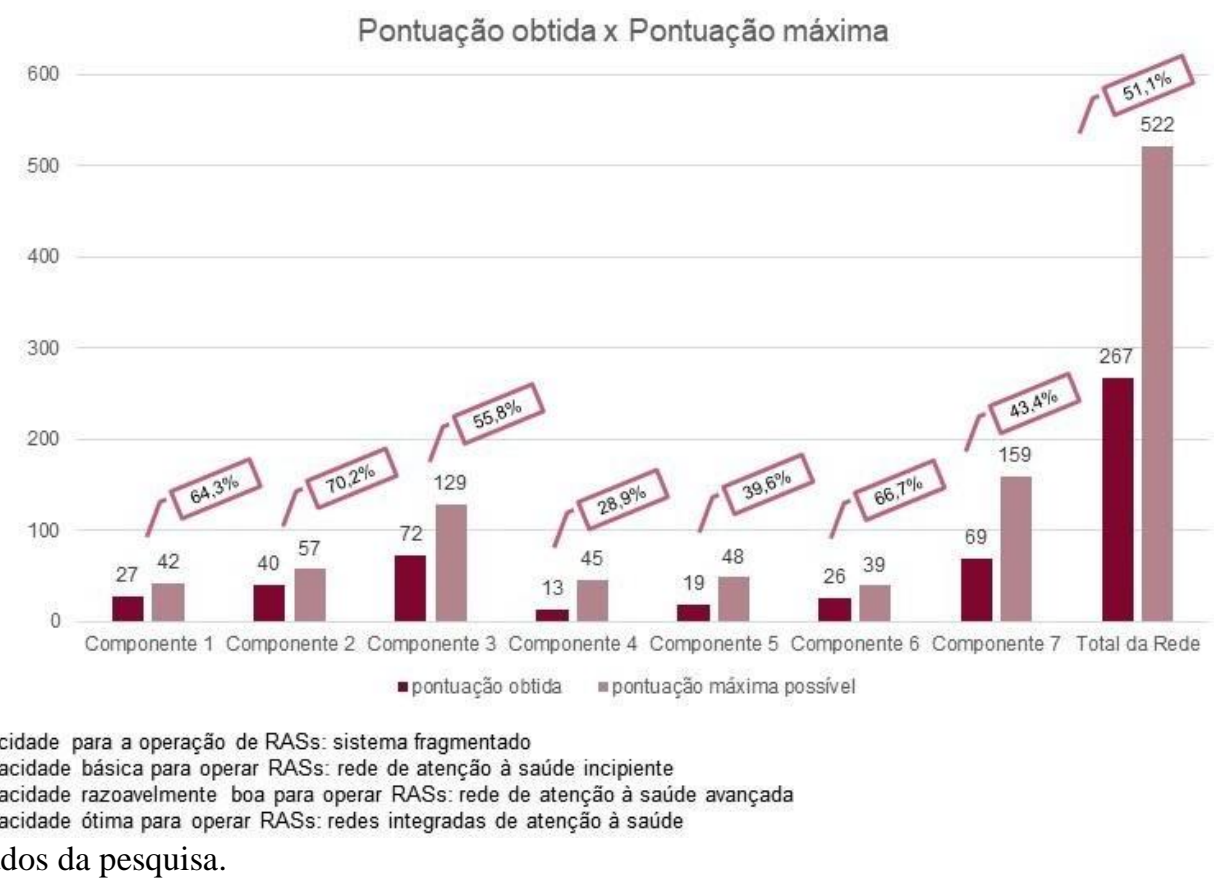

A pontuação total do diagnóstico da Rede Municipal de saúde de Macaé, traduzido pelo instrumento foi de 267 pontos, o que de acordo com a análise do escore utilizado aponta para uma Rede de Atenção à saúde avançada, com uma capacidade boa para operar a Rede.

Demonstra-se ainda a seguir, na Figura 2, um panorama visual da distribuição do quantitativo de questões que representaram os principais nós identificados a partir do instrumento aplicado: 
Figura 2 - Nós críticos- Pontuações 0 e 1

\section{Nós CRíTICOS -}

\section{PONTUAÇÕES 0 E 1}

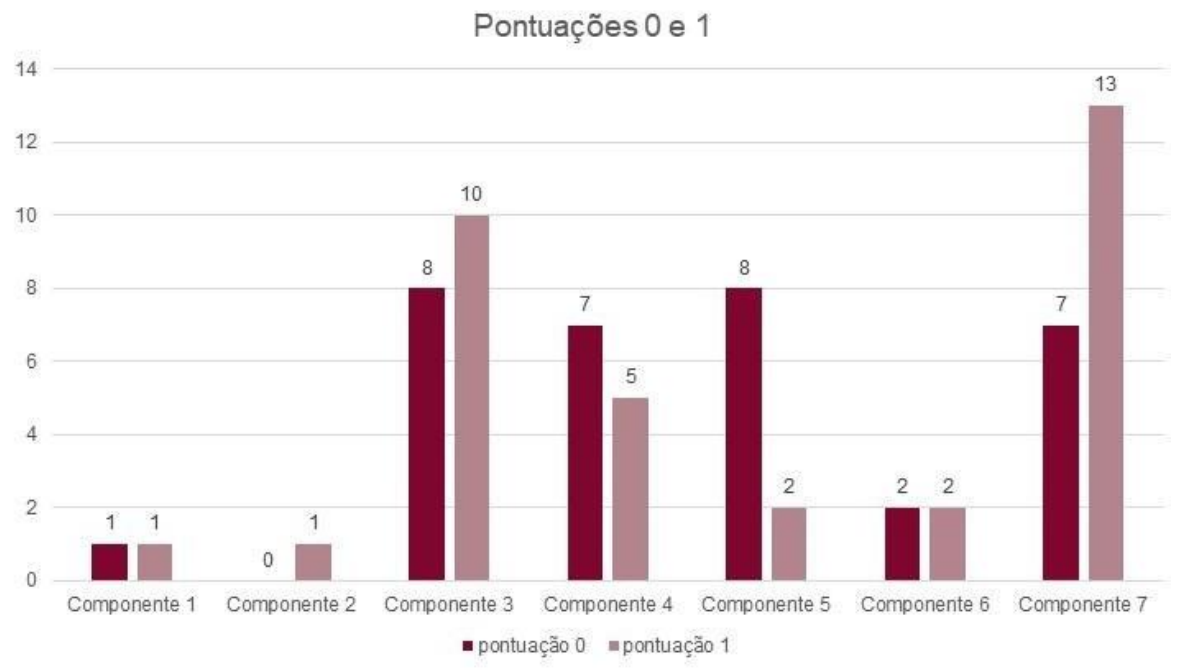

0 (zero): corresponde à não existência do que está sendo perguntado;

1 (um): existe o que está sendo perguntado, mas com um funcionamento básico ou incipiente;

Fonte: Dados da pesquisa.

Observa-se por meio dos resultados já apresentados, que os componentes 4 e 5, são os que apresentam menores percentuais de alcance da pontuação máxima desejada com 28,9\% e 39,6\% respectivamente. E os melhores percentuais de pontuação atingida foram evidenciados nos componentes 2 e 6, com 70,2\% e 66,7\% do total máximo respectivamente, conforme pode-se observar na Figura 3, que demonstra o quantitativo de questões que pontuaram com escore 2 e 3 dentro de cada um dos componentes avaliados: 
Figura 3 - Pontuações 2 e 3

\section{PONTUAÇÕES 2 E 3}

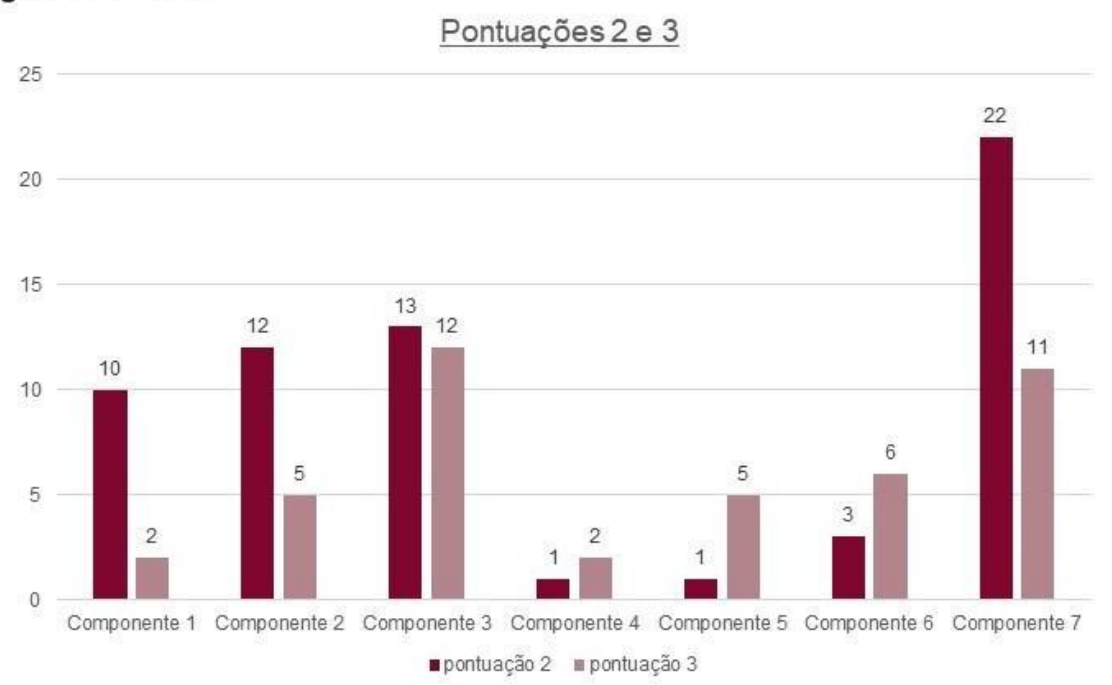

2 (dois): existe o que está sendo perguntado, com um funcionamento razoavelmente bom, mas insuficiente;

3 (três): corresponde à sua existência em operação de forma ótima.

Fonte: Dados da pesquisa.

Apesar do destaque positivo para o componente referente à APS, nos espaços destinados a comentários ao final dos formulários, dois voluntários preencheram com apontamentos referentes a necessidade do fortalecimento da APS no município no sentido de ser ordenadora da Rede e como meio para mudança de modelo de atenção à saúde da cidade, caracterizado então como hospitalocêntrico.

\section{DISCUSSÃO}

Com relação ao modelo de atenção à saúde adotado pelo município ser marcadamente um modelo hospitalocêntrico conforme indicado no espaço de comentários do instrumento, essa caracterização é também corroborada quando em um estudo sobre a cidade de Macaé, ao constatarse elevados óbitos por doenças do aparelho circulatório, aponta-se a necessidade de uma reavaliação estrutural da APS, o que perpassa o modelo assistencial e a ampliação da Estratégia Saúde da Família (ESF) no território municipal (LOURENÇO et al., 2019).

No contexto da boa pontuação, em geral, acerca da oferta de leitos e serviços hospitalares, salienta-se que a cidade teve um aumento de leitos nos últimos anos, sendo em 2012 
contabilizados 405 leitos SUS, e em 2017: 438 leitos e além disso, a rede pública, com destaque para o Hospital Público de Macaé concentra a maioria dos serviços de urgência e emergência, ainda que $43 \%$ da população municipal seja coberta por planos de saúde (LOURENÇO et al, 2019).

No aspecto dos desafios apresentados pela rede municipal, demonstrou-se por diversos elementos a fragilidade no estabelecimento de proximidade entre os serviços ambulatoriais e hospitalares com a APS. Salienta-se aqui que uma parte dos nós críticos apresentados nesse sentido, transversalizaram o fato da não existência de prontuário eletrônico funcionante na APS durante o período de coleta de dados da presente pesquisa. Período este em que o município estava em processo de organização e garantia dos meios para a informatização da rede básica de saúde, o que pode refletir mudanças significativas em futuros estudos, principalmente quanto ao aspecto de adscrição da população e gerenciamento dos cadastros por unidade familiar, e outras dimensões avaliadas, naquele momento, de forma negativa.

Ainda no contexto dos desafios foi evidenciada a necessidade de fortalecimento e organização dos serviços de forma a haver clareza nos papéis dos diferentes níveis de atenção, e objetivos comuns, permitindo que as equipes de APS possam saber identificar em que situações os usuários precisam ser encaminhados a outros pontos de atenção da Rede.

Nesse sentido, um estudo sobre o uso da Referência e contrarreferência na APS de um município do estado de Minas Gerais apontou alguns fatores negativos relacionados ao acesso aos serviços de saúde, presentes no cotidiano da APS, tais como: “encaminhamentos desnecessários, ações médicas pouco resolutivas no nível primário, contrarreferência pouco frequente, falta de recursos e estruturas, investimentos insuficientes" (MELLO et al. 2016, p. 4994). Esses elementos certamente refletem parte da realidade de muitos outros municípios do país, onde a integração entre os diversos serviços da rede de saúde não se dá de modo adequado, inviabilizando a consolidação da resolubilidade enquanto um princípio organizativo do SUS.

A existência dessa proximidade entre os diversos serviços da Rede com uma articulação clara quanto aos critérios e o modo de encaminhamento dos usuários atendidos, certamente permitiria o alcance de maior qualidade no nível do acompanhamento de saúde da população e maior resolutividade da APS.

Ressalta-se ainda que uma revisão de escopo recente sobre o conceito de rede de atenção à saúde e suas características-chave, demonstrou dentre os seus resultados a ausência de um conceito totalizante capaz de traduzi-la na sua total abrangência e significado, entretanto, termos como 
articulação, fluidez, integração e interligação permeiam elementos presentes nos conceitos mapeados (NAKATA et al. 2020). Essas características, podem ser traduzidas no presente estudo como imagens-objetivos em direção ao qual precisa-se avançar em termos de planejamento e gestão do sistema municipal de saúde avaliado quantos aos meios necessários para alcançá-las. Como garantir, por exemplo, que haja fluidez nos processos de comunicação instituídos entre os diferentes níveis de atenção? Como instituir fluxos que articulem necessariamente processos de trabalho produtores de cuidado que tragam centralidade ao usuário? Como integrar e interligar profissionais da saúde demais setores que se associam a ela, de modo a garantir a continuidade de cuidado para os usuários assistidos pela rede?

Sendo assim, defende-se que o caminho para a melhoria da integração da Rede de atenção à saúde a nível municipal perpassa necessariamente pela criação de mecanismos de aproximação entre os serviços dos diferentes níveis de atenção. Seja através do uso de sistemas de informação que possam gerenciar a movimentação dos usuários nos diferentes níveis de atenção, ou seja, através da criação de espaços institucionais que permitam o diálogo entre as diferentes linhas de cuidado. Como, por exemplo, um fórum permanente que agregue profissionais envolvidos na gestão e na assistência à saúde da gestante no município, onde houvesse discussão de casos, elaboração protocolos claros quanto à articulação das diferentes unidades envolvidas no processo de acompanhamento dessas gestantes, bem como debates acerca de novas evidências que possam contribuir para melhoria da qualidade da assistência à saúde da gestante no município. Destaca- se que este caminho também poderia contribuir para a melhoria do desafio apontado nos resultados como dificuldade de articulação com a maternidade e ambulatório de acompanhamento de alto risco.

A ideia da criação de espaços institucionais de diálogo entre os diversos serviços poderia ser aplicada a quaisquer das linhas-guia existentes. Seja das doenças infecciosas às doenças crônicas, da criança ao idoso, além de viabilizar a avaliação e revisão periódica das mesmas- ponto esse, também evidenciado como fragilidades da rede.

Outro ponto que soma em favor de estratégias construídas nesse sentido, é que $75 \%$ dos servidores distribuídos nos 81 estabelecimentos de saúde pública municipal em Macaé, são concursados, o que favorece a continuidade de iniciativas e impactos positivos de investimento em recursos humanos para melhoria das práticas assistenciais (PASSOS; BARBOSA, 2019). 
A formalização de medidas que garantam o reconhecimento do papel das diversas unidades e profissionais que compõem organicamente essa Rede, certamente será de grande valia para o alcance de uma Rede mais integrada e consequentemente, para melhoria da qualidade da atenção à saúde da população.

\section{CONCLUSÃO}

O diagnóstico da Rede de Atenção à Saúde no município de Macaé apontou para uma Rede avançada, com uma boa capacidade operativa, evidenciando fragilidades que dentre muitos aspectos estavam atreladas também à capacidade de articulação entre os diferentes níveis assistenciais e ao uso de tecnologias de informação. Nesse sentido, um novo estudo poderia apontar um cenário diferenciado, uma vez que o município passava por processo de informatização da APS no período de coleta de dados.

Enquanto limitação da presente pesquisa aponta-se o potencial de generalização dos dados, por se tratar de uma realidade particular do município estudado. Entretanto, entende-se que do ponto de vista de um sistema de saúde como o SUS que tem na descentralização de forma municipalizada dos serviços, o eixo de organização político- administrativa focal das últimas décadas, que estudos como este, podem trazer à tona desafios semelhantes em distintas realidades do país. Fortalecendo nesse sentido, o impulsionamento de novas pesquisas que possam contribuir com o planejamento e a gestão pública da saúde num país de organização federativa como o Brasil, onde olhar para a operacionalização das macropolíticas que efetivam direitos sociais como o caso da saúde é tão importante quanto compreender os percalços da sua concretização a nível local.

\section{REFERÊNCIAS}

\section{BRASIL. Portaria $N^{\circ}$ 3, de 28 de setembro de 2017. Consolidação das normas sobre redes do sistema Único de saúde. Disponível em: http://bvsms.saude.gov.br/bvs/saudelegis/gm/2017/prc0003_03_10_2017.html. Acesso em: 18 mar. 2021.}


BRASIL. Presidência da República. Casa civil. Subchefia para assuntos jurídicos. Decreto 7508 de 28 de junho de 2011. Regulamenta a Lei $n^{0}$ 8.080, de 19 de setembro de 1990, para dispor sobre a organização do Sistema Único de Saúde - SUS, o planejamento da saúde, a assistência à saúde e a articulação interfederativa, e dá outras providências. Brasília, 2011. Disponível em: http://www.planalto.gov.br/ccivil_03/_ato2011-2014/2011/decreto/d7508.htm. Acesso em: 10 mar. 2021.

KUSCHNIR, Rosana; CHORNY, Adolfo Horácio. Redes de atenção à saúde: contextualizando o debate. Revevista Ciência \& saúde coletiva. [Internet]. Rio de Janeiro, v. 15, n. 5, p. 2307-2316. 2010. Disponível em: http://www.scielo.br/scielo.php?script=

sci_arttext\&pid=S1413-81232010000500006\&lng=pt\&nrm=iso. Acesso em: 11 abr. 2021.

LEAL, Daniele L.; WERNECK, Marcos A. F.; BORGES-OLIVEIRA, Ana Cristina. Validação da versão saúde bucal do Instrumento de Diagnóstico do Estágio de Desenvolvimento da Rede de Atenção à Saúde. Revista Pan-Amazônica de Saúde. [Internet]. v. 8, n. 4, p. 11-11. 2017. Disponível em: http://scielo.iec.gov.br/pdf/rpas/ v8n4/2176-6223-rpas-8-04-00065.pdf. Acesso em: 11 abr. 2021.

LOURENÇO, Ana Luiza P. et al. Trajetória da Atenção à saúde em Macaé: Desafios e perspectivas. In: SILVA, Scheila R.; CARVALHO, Meynardo R. (Org.). Macaé, do caos ao conhecimento: olhares acadêmicos sobre o cenário de crise econômica. Macaé: Prefeitura Municipal de Macaé. 2019. p. 386-396.

MELO, Duane F.; CRISCUOLO, Maria B. R.; VIEGAS, Selma M. da F. Referência e contrarreferência no cotidiano da atenção à saúde de Divinópolis-MG, Brasil: o suporte às decisões da atenção primária. Revista de Pesquisa: Cuidado é Fundamental Online. [Internet]. v. 8 , n. 4, p. 4986-4995. 2016. Disponível em: http://www.seer.unirio.br/cuidadofundamental/article/view/4402/pdf_1. Acesso em: 11 abr. 2021. MENDES, Eugênio V. As redes de atenção à saúde. 2. ed. Brasília-DF: Organização PanAmericana da Saúde, 2011.

NAKATA, Liliane C. et al. Conceito de rede de atenção à saúde e suas características-chaves: uma revisão de escopo. Rev. Escola Anna Nery, [Internet]. v. 24, n. 2, p. 1-12. 2020. Disponível em: http://www.revenf.bvs.br/scielo.php?script=sci_arttext\&pid=S14 14-81452020000200701. Acesso em: 11 abr. 2021.

ORGANIZAÇÃO PAN-AMERICANA DE SAÚDE. Informe Dawson sobre el futuro de los servicios médicos y afines. Washington, DC: Pan American Health Organization, 1964. Disponível em: https://iris.paho.org/handle/10665.2/1133. Acesso em: 30 mar. 2021.

ORGANIZAÇÃO MUNDIAL DE SAÚDE (OMS). The world health report 2008: primary health care now more than ever. World Health Organization, 2008.

PASSOS, Elaine A. A.; BARBOSA, Sabrina N. D. Saúde em Macaé: Cenário pré e pós crise e seus determinantes para a sociedade. In: SILVA, Scheila R.; CARVALHO, Meynardo R. (Org.). Macaé, do caos ao conhecimento: olhares acadêmicos sobre o cenário de crise econômica. Macaé: Prefeitura Municipal de Macaé. 2019. p. 365-384. 
RODRIGUES, Ludmila B. B. et al. Coordenação das redes de atenção à saúde pela atenção primária: validação semântica de um instrumento adaptado. Revista Cadernos de Saúde Pública. [Internet]. v. 30, n. 7, p. 1385-1390. 2014. Disponível em: https://www.scielo.br/pdf/csp/v30n7/0102-311X-csp-30-7-1385.pdf. Acesso em: 11 abr. 2021.

SCHILLER, Carolina O. A. et al. Validação de face e construto do Instrumento de Avaliação de Redes de Atenção Materno-infantil (IARAMI). Revista Ciência \& saúde coletiva. [Internet]. 2020. Disponível em: http://www.cienciaesaudecoletiva.com.br/ artigos/validacao-de-face-e-construto-do-instrumento-de-avaliacao-de-redes-de-atencaomaternoinfantil-iarami/17505?id=17505\&id=17505. Acesso em: 11 abr. 2021.

VIANA, Ana Luiza et al. Regionalização e redes de saúde. Revista Ciência \& saúde coletiva. [Internet]. v. 23, p. 1791-1798. 2018. Disponível em: http://www.scielo.br/ scielo.php?pid=S1413-81232018000601791\&script=sci_abstract\&tlng=pt. Acesso em: 10 mar. 2021. 